\title{
COVID-19 Related Racial Discrimination in Small Asian Communities: A Cross Sectional Study
}

\author{
Minji Kim ${ }^{1}\left[\right.$ Seiya Liu ${ }^{1} \cdot$ Yonghoon Lee $^{1} \cdot$ Cho-Hee Shrader $^{2} \cdot$ Mariano Kanamori $^{2}$
}

Accepted: 10 October 2021 / Published online: 16 October 2021

(c) The Author(s), under exclusive licence to Springer Science+Business Media, LLC, part of Springer Nature 2021

\begin{abstract}
Since the outbreak of COVID-19, there has been a surge of discrimination against Asians across the globe. However, there is a knowledge gap of COVID-19 related racial discrimination against Asians in smaller Asian populations. A total of 221 adults living in Florida completed an online survey between June-July 2020. Adjusted logistic regression assessed associations between sociodemographic factors and experienced discrimination, hypervigilance of safety, nervousness in public, and anticipated discrimination. Statistical analyses were performed using SPSS. Asian respondents were more likely than non-Asians to experience discrimination during COVID-19 (AOR $=12.58 ; 95 \% \mathrm{CI} 4.74,33.38 ; \mathrm{p} \leq 0.001)$. Asians were more likely to anticipate discrimination after the pandemic ends ( $\mathrm{AOR}=4.35,95 \% \mathrm{CI} 1.33,14.17 ; \mathrm{p}<0.05)$. We found that Asians in smaller Asian populations suffer from a disproportionate level of discrimination due to COVID-19, relative to non-Asians. Our findings support previous research that racial discrimination exists on a continuum of violence and can have severe negative health consequences.
\end{abstract}

Keywords COVID-19 $\cdot$ Pandemic $\cdot$ Stigma $\cdot$ Asian $\cdot$ Discrimination $\cdot$ Racism

\section{Background}

In March 2020, the World Health Organization (WHO) declared the novel COVID-19 outbreak as a pandemic [1, 2]. The pandemic which was traced to Wuhan, China, has caused devastating effects throughout the world and has impacted virtually all races and ethnicities; yet, some media propaganda in the United States (U.S.) have reported the virus as the "China Virus," "Kung flu," and "China virus pandemonium" [3]. Shortly after the US media's association of COVID-19 with China, hate crimes against all Asians subgroups across the country drastically increased [2]. For example, there were over 1135 reports of COVID-19-related hate crimes and discrimination against Asians within the first 2 weeks of launching the STOP AAPI Hate crime incident reporting platform on March 19, 2020 [4]. In Midland,

Minji Kim

minji.kim@med.miami.edu

1 University of Miami Miller School of Medicine, 1600 NW 10th Ave \#1140, Miami, FL 33136, USA

2 Division of Prevention Science and Community Health, Department of Public Health Sciences, University of Miami Miller School of Medicine, Miami 33136, USA
Texas, there was an attempted murder of a Burmese-American family at a Sam's Club for being Chinese and "infecting people with China Virus" [5]. Another study found that $30 \%$ of Americans blamed China and/or Chinese people for the COVID-19 pandemic, and one in three respondents witnessed someone blaming Asians for the pandemic [6]. In response to the surge of hate crimes, over 450 civil rights, social justice, and labor organizations called on Congress to denounce the hostile rhetoric against the Asian-American community [7].

Experienced discrimination can have negative long-term effects on Asian health outcomes, especially in regions where Asians are minorities and considered more 'foreign,' such as Florida [8]. Experienced discrimination is defined as the experience of stigmatizing behaviors from others. Exposure to racial or ethnic discrimination is a chronic source of trauma, resulting in long-term mental and physical disorders, such as cardiovascular disease [8-10]. Such correlation was observed in Muslims after the 9/11/2001 attacks on the U.S. World Trade Center. Muslims reported poorer mental health, scoring higher on depressive and PTSD symptoms than nonMuslim counterparts [11]. Furthermore, anticipated discrimination is defined as expectations of bias being perpetrated by others. Anticipated discrimination is also associated 
with increased depressive distress and cardiovascular stress responses, as demonstrated by studies among other stigmatized communities [12-14]. Anticipated discrimination can also contribute to an avoidance of accessing health services, leading to negative health outcomes [15]. Thus, examining racial discrimination is critical in recognizing and preventing the potential physical and mental harm it can cause.

Research indicates that over half of the reported hate crimes during the COVID-19 pandemic occurred in California and New York (58.5\%), where Asians make up 14.7\% and $8.5 \%$ of the population, respectively $[16,17]$. On the other hand, only $1.6 \%$ of hate crimes were reported from Florida [16]. This may be due to a small number of Asians in Florida, as only Asians compose $2.8 \%$ of the state's population, or due to potential underreporting of hate crimes. Florida's racial distribution is mostly comprised of White, Hispanic, and Black individuals, respectively [16-18]. This paper operationalizes a "small Asian community" as a community in which less than $5.7 \%$ of the population is Asian, as labelled by U.S. Census Bureau in 2017 [19]. One paper in the 1980s examined the incidence of coronary abnormalities in a "small Asian population," where only $1.7 \%$ of the study area population was Asian [20]. Given that the Asian population in the U.S. quadrupled from 1980 to 2010 [21], we assessed Florida as having "small Asian communities." Discrimination in small Asian communities may be overlooked, underreported, and even more extreme than in communities with more Asians [18]. Thus, the current paper seeks to examine the impact of COVID-19 on the experienced discrimination of Asians in Florida. We hypothesized that Asians would experience more discrimination, be more hypervigilant, and be more nervous during the pandemic, and anticipate more discrimination after the pandemic ends, relative to non-Asians. To the best of our knowledge, this is the first paper to explore discrimination against Asians in small Asian communities during the COVID-19 pandemic.

\section{Theoretical/Conceptual Framework}

This study is guided by The Health Stigma and Discrimination Framework to assess discrimination among this population and to understand health impacts [12]. This approach describes how racial stigma intersects with health-related stigmas and leads to social and health impacts. There are factors that drive the health-related stigma, and the stigma 'marking,' in which stigma is applied to people according to a specific health condition or other differences like race and occupation. Stigma manifests in a range of experiences and practices, including experienced stigma and anticipated stigma. An example of experienced stigma is the feeling one has after being unreasonably rejected in a job application whereas an example of anticipated stigma is when an individual does not apply for a job because she/he expects to be rejected $[12,14]$. For the purpose of this study, hypervigilance of safety and nervousness were added because previous studies have reported an association between racial harassment and hypervigilance among minorities, and discrimination and anxiety among Asian-Americans [22, 23]. The Health Stigma and Discrimination Framework guided us to examine these factors in the present study.

\section{Methods}

\section{Participants and Data Collection}

Data were collected through an online survey on Qualtrics (Qualtrics, Provo, UT) for three weeks between June and July 2020. There were 56 items on the survey. Recruitment occurred using respondent-driven sampling of popular opinion leaders and snowball sampling as is commonly used in cross-sectional internet based surveys targeting minority populations [24]. Accordingly, study staff emailed the survey to community leaders throughout Florida and requested they distribute the survey to their social networks. The survey was also available on social media platforms and sent out to various schools and organizations in order to recruit nonAsian respondents. The survey was available in languages common in Florida in order to include as many participants as possible: English, Spanish, Chinese (Simplified), Korean, Vietnamese, and Haitian Creole [25]. The survey was written in English, translated to each of these languages, then back-translated by a certified bilingual native translator [26]. Informed consent to participate in the study was obtained from all participants. Participant inclusion criteria included: (a) being age 18 years or older, and (b) living in Florida during the pandemic (January 2020 to the time of study participation). This study was reviewed and approved by the University of Miami's Institutional Review Board and adhered to the tenets of the Declaration of Helsinki. The data was stored on a university secured data network.

\section{Measures}

Demographic and the experienced discrimination outcomes were both adapted from the "Mt. Sinai Speak UP on COVID-19 Survey," a survey that examined participants' COVID-19-related physical and mental health, social determinants, and attitudes [27]. We also adapted STOP AAPI's hate crime reporting form to assess reason and type of discrimination [28]. Non-Asians are those who did not identify themselves as Asian. Demographic characteristics included categorical measures of age categories in years (18-24 as referent group, 25-34, 35-44, 45-54, 55-64, 65+), gender (male as referent group, female, non-binary), sexuality 
(heterosexual as referent, homosexual), race (non-Asian as referent group, Asian), country of birth (not born in the U.S. as referent, born in U.S), education (no college degree as referent, college degree), employment (full-time employment as referent, not full-time employment), and income (less than $\$ 75,000$ as referent, more than $\$ 75,000$ ). Continuous measures included length of stay in Florida in years. Respondents also reported their work situation during the pandemic (not working or working less as referent, working the same as or more than before the pandemic), and perceived number of Asians in their community (no Asians in community as referent, a few Asians in their community, a lot of Asians in their community).

The experienced discrimination measure asks participants to indicate the extent to which they have felt discriminated against during the pandemic. The anticipated discrimination measure asks participants to indicate the extent to which they expect to be discriminated against after the pandemic ends. The outcome variable of experienced discrimination and anticipated discrimination were coded as binary for 'Not at all,' and 'Less than' $=0$, and 'The same as,' and 'More than before the pandemic' $=1$. Participants who reported experiencing discrimination were prompted with additional survey questions regarding the reason and type of discrimination. Hypervigilance of personal safety and nervousness of experiencing a hate crime in public spaces during the pandemic were ranked using a 5-point Likert scale $(1=$ strongly disagree and $5=$ strongly agree).

\section{Qualitative}

Participants who reported that they felt discriminated against 'The same as' or 'More than before than pandemic' were asked to follow-up with additional details of the experienced discrimination. Responses were translated to English if reported in another language. The qualitative data was not coded for this study.

\section{Statistical Analysis}

Binary logistic regression models were used to assess associations between Asians versus non-Asians with sociodemographic characteristics, experienced discrimination, hypervigilance, nervousness, and anticipated discrimination

Table 1 Socio-demographic characteristics

\begin{tabular}{|c|c|c|c|}
\hline & Asians $(\mathrm{n}=168)$ & Non-Asian $(\mathrm{n}=53)$ & Total sample $(\mathrm{n}=221)$ \\
\hline \multicolumn{4}{|l|}{ Age } \\
\hline $18-24$ & $20.2 \%$ & $52.8 \% *$ & $28.1 \%$ \\
\hline $25-34$ & $25.0 \%$ & $30.2 \%$ & $26.2 \%$ \\
\hline $35-44$ & $15.5 \%$ & $3.8 \%$ & $12.7 \%$ \\
\hline $45-54$ & $17.9 \%$ & $9.4 \%$ & $15.8 \%$ \\
\hline $55-64$ & $16.7 \%$ & $1.9 \%$ & $13.1 \%$ \\
\hline $65+$ & $4.8 \%$ & $1.9 \%$ & $4.1 \%$ \\
\hline Sexuality (heterosexual) & $86.3 \%$ & $79.2 \%$ & $84.6 \%$ \\
\hline Gender (male) & $32.7 \%$ & $30.2 \%$ & $32.1 \%$ \\
\hline \multicolumn{4}{|l|}{ Asians in community } \\
\hline None & $8.3 \%$ & $11.3 \%$ & $9.0 \%$ \\
\hline Few & $75.0 \%$ & $71.7 \%$ & $74.2 \%$ \\
\hline A lot & $16.7 \%$ & $17.0 \%$ & $16.7 \%$ \\
\hline Length of stay in FL (in years, mean \pm SD) & $14.6 \pm 11.8$ & $13.7 \pm 10.5$ & $14.4 \pm 11.5$ \\
\hline Born in USA (yes) & $22.6 \%$ & $75.5 \% *$ & $35.3 \%$ \\
\hline Education ( > graduated from college) & $85.1 \%$ & $86.8 \%$ & $85.5 \%$ \\
\hline Employment (full-time) & $45.8 \%$ & $26.4 \% *$ & $41.2 \%$ \\
\hline Income $(>\$ 75,000)$ & $31.5 \%$ & $39.6 \%$ & $33.5 \%$ \\
\hline Not working/working less in COVID & $46.4 \%$ & $30.2 \% *$ & $42.5 \%$ \\
\hline Completed the survey in an Asian language & $41.1 \%$ & $1.9 \% *$ & $31.7 \%$ \\
\hline Experienced discrimination (the same as or more than) & $56.5 \%$ & $18.9 \% *$ & $47.5 \%$ \\
\hline Anticipated discrimination (the same as or more than) & $63.1 \%$ & $28.3 \% *$ & $54.8 \%$ \\
\hline Hypervigilance (agree or strongly agree) & $58.4 \%$ & $9.5 \% *$ & $46.6 \%$ \\
\hline Nervousness (agree or strongly agree) & $41.0 \%$ & $7.6 \% *$ & $33.0 \%$ \\
\hline
\end{tabular}

$S D$ standard deviation

$* \mathrm{p}<0.05$ : significant difference between Asians and non-Asians 
(Table 1). Adjusted odds ratio (AOR), 95\% confidence intervals (CI) and the p-values were calculated using SPSS Statistics (Version 26.0.0, 2019, Armonk, NY).

\section{Results}

Table 1 describes the socio-demographic information of respondents. Of 168 Asian and 53 non-Asian participants, the majority were between ages 18 to 45 years $(60.7 \%$ of Asians and $86.8 \%$ of non-Asians). Of the non-Asians, $66.7 \%$ were White, $21.1 \%$ were Hispanic, $19.3 \%$ were Black, and $7.1 \%$ also identified as Other. Participants were allowed to check on more than one racial subgroup. While $75.5 \%$ of non-Asians were born in the U.S., only $22.6 \%$ of Asian respondents were born in the U.S. Of the $77.4 \%$ Asians born outside of the U.S., the majority of respondents were born in China $(\mathrm{n}=88)$ and South Korea $(\mathrm{n}=17)$. Furthermore, $46.4 \%$ of Asian respondents and $30.2 \%$ of the non-Asian reported not working or working less during the COVID19 pandemic. The majority of participants reported living in communities with 'no or few' Asians (83.2\%), and only $16.7 \%$ in communities with 'a lot' of Asians.

\section{Experienced Discrimination}

As seen in Table 2, respondents who were between the ages 45 to 54 years $(\mathrm{AOR}=0.24 ; 95 \% \mathrm{CI} 0.07,0.83 ; \mathrm{p}<0.05)$ and ages 65 years and older $(\mathrm{AOR}=0.04,95 \% \mathrm{CI} 0.004$, $0.44 ; \mathrm{p}<0.05$ ) were less likely to report experienced discrimination than respondents who were between ages 18 to 24 years. Asian respondents were more likely to report experienced discrimination during the COVID-19 pandemic relative to non-Asians $(\mathrm{AOR}=12.58$; $95 \% \mathrm{CI} 4.74,33.38$; $\mathrm{p} \leq 0.001$ ). Distribution of responses for experienced discrimination between Asians and non-Asians is shown in Fig. 1. There was no statistical difference in experienced discrimination between non-Chinese Asians and Chinese Asians. There was no statistical difference in the length of stay in Florida and experienced discrimination. Of the 95 Asian participants who reported experiencing discrimination during the pandemic, 86 respondents indicated the reason(s) for discrimination $-94.2 \%$ believed it was due to racial profiling and $36.0 \%$ believed wearing a mask to be the cause. Others believed it was due to their gender (4.7\%), age $(4.7 \%)$, or other $(16.4 \%)$, such as, sexuality, religion, education, clothing, job, and home location. Furthermore, of 82 Asian respondents who indicated the type of discrimination, $43.9 \%$ reported shunning and $41.5 \%$ reported being verbally harassed. Some participants $(15.9 \%)$ reported that their discrimination was through social media platforms and $12.2 \%$ reported that it was a workplace discrimination. A quarter $(25.5 \%)$ reported that they were either cough or spat on, barred from establishment, or experienced vandalism.

\section{Hypervigilance During COVID-19}

More than half of Asian respondents (58.4\%) reported being hypervigilant of their personal safety due to their race/ethnicity during COVID-19 (Fig. 2). As shown in Table 2, participants who experienced discrimination during COVID-19 were more likely to report hypervigilance for their safety than those who have not experienced discrimination, regardless of their race (AOR $=1.79 ; 95 \%$ CI 1.01, 3.19; $\mathrm{p}<0.05$ ). Compared to non-Asians, Asians were more likely to report hypervigilance for their safety $(\mathrm{AOR}=11.03 ; 95 \%$ CI 4.83, 25.23; $\mathrm{p}<0.001)$.

\section{Nervousness During COVID-19}

Similarly, $41.0 \%$ of Asian participants reported being nervous/scared to go to public place because they may experience discrimination due to their race/ethnicity (Fig. 2). They were also more likely to report that they were nervous/scared to go outside to public places because of fear of experiencing discrimination due to their race/ ethnicity than non-Asians (AOR $=3.03 ; 95 \%$ CI 1.68 , 5.47; $\mathrm{p}<0.05)$. Those who completed the survey in an Asian language were more likely to indicate that they were nervous/scared than those who completed the survey in a non-Asian language $(\mathrm{AOR}=2.56 ; 95 \%$ CI 1.25, 5.24; $\mathrm{p}<0.05)$.

\section{Anticipated Discrimination}

As shown in Fig. 1, 63.7\% of Asian participants and 28.3\% of non-Asian participants reported that they anticipate similar or higher levels of discrimination after the pandemic ends. Respondents who reported experienced discrimination during the pandemic were more likely to report that they anticipate similar or a higher level of discrimination after the pandemic ends than those who did not experience racial discrimination $(\mathrm{AOR}=29.36$; 95\% CI 11.54, 74.73; $\mathrm{p}<0.001$; Table 2). Respondents who were ages 55-64 years were less likely to report similar or a higher level of discrimination than other age groups post-pandemic ( $\mathrm{AOR}=0.12 ; 95 \% \mathrm{CI}$ $0.02,0.70 ; \mathrm{p}<0.05)$. Respondents who reported working the same or more during the pandemic than before were more likely to report that they anticipate similar or higher level of discrimination after the pandemic $(\mathrm{AOR}=2.55 ; 95 \% \mathrm{CI}$ $1.04,6.38 ; \mathrm{p}<0.05)$. Asian respondents were more likely to report that they anticipate similar or higher level of discrimination after the pandemic than non-Asians $(\mathrm{AOR}=4.35$, 


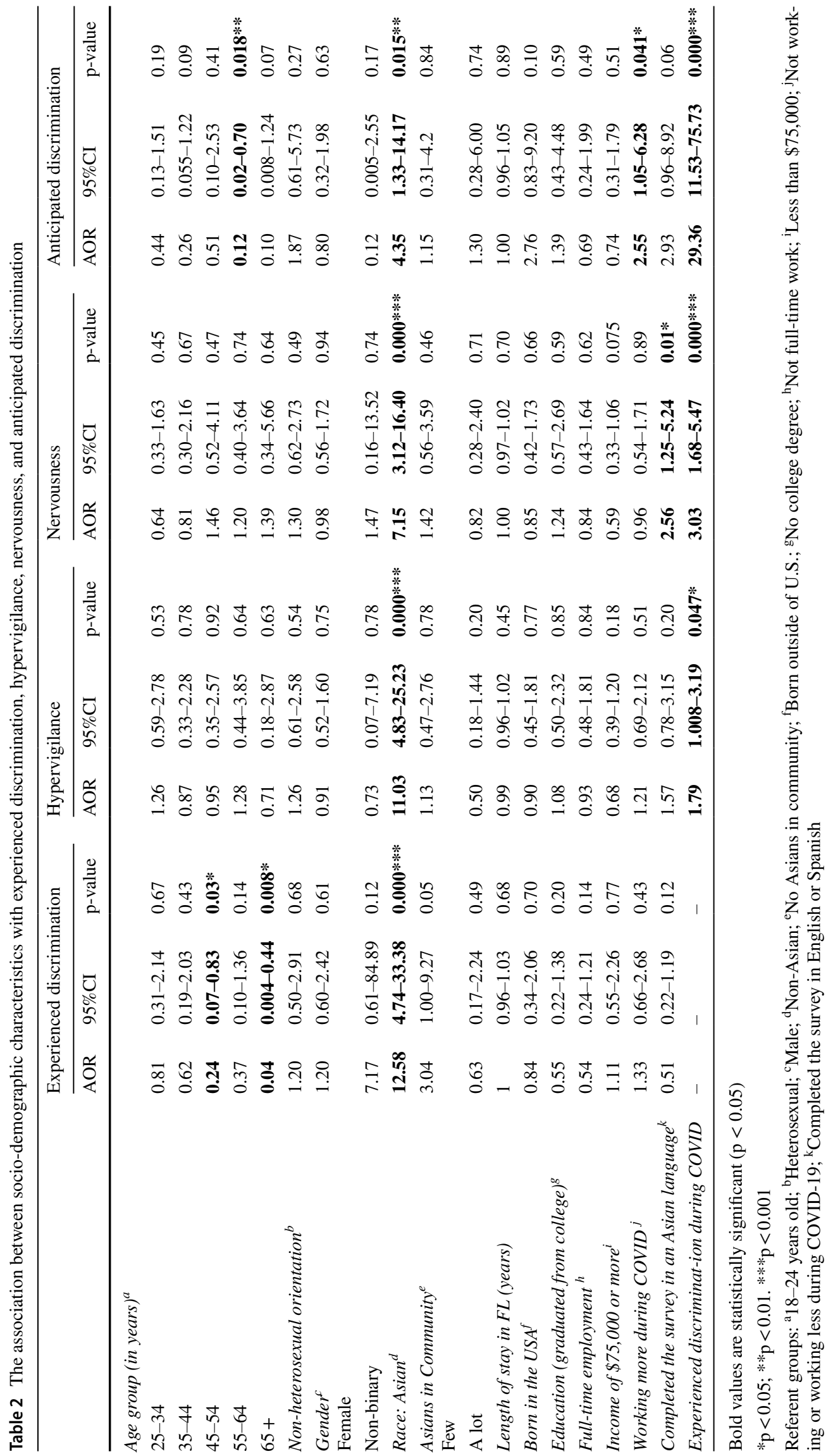


Fig. 1 Distribution of responses for experienced and anticipated discrimination

\section{Experienced and Anticipated Discrimination between Asians and non-Asians}

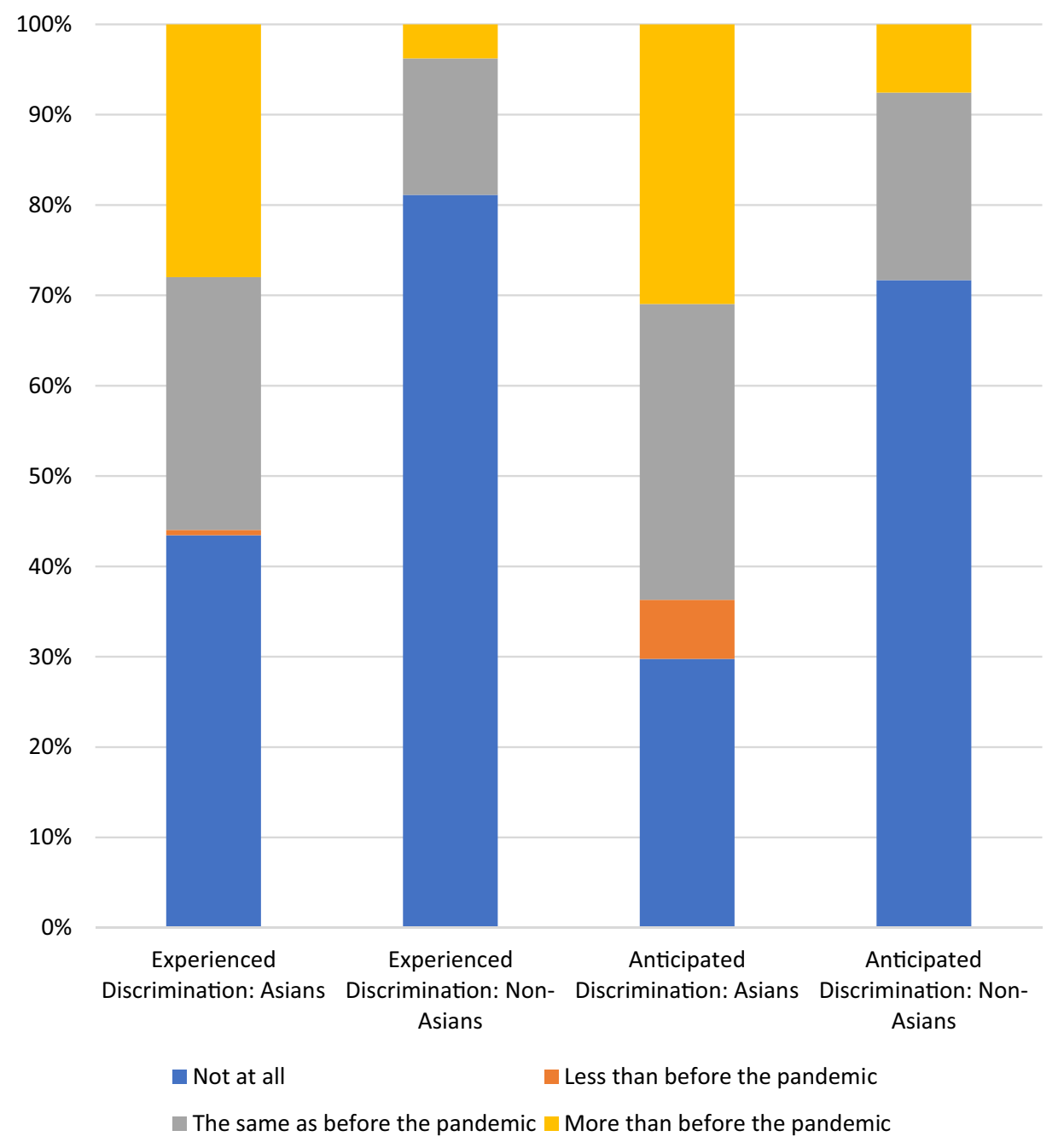

95\% CI 1.33, 14.17; p <0.05). Lastly, there was no statistical difference in anticipated similar or higher level of discrimination between non-Chinese Asians and Chinese Asians.

\section{Qualitative Findings}

Of the Asians who experienced racial discrimination during COVID-19, 39 participants described their racial encounter. One description was excluded because it was not a racial discrimination; the individual described that she or he had to quarantine after returning to Miami. There were 11 male and 27 female participants. Most of the experiences (20) were verbal harassment. Of these, seven participants were referred to as "China Virus" and 13 were verbally attacked. For example, the respondents shared that, "two men saw me wear a mask and shouted, "Chinese Virus" and "I was told that the virus is in Chinese blood." Those who were verbally attacked were told "Go back to where you came from." One participant shared that she or he felt like an 'outsider' when trying to enter an open community: "A security guard... suddenly shouted at me, 'Hey Mandarin! Mandarin! The [Brickell key] island is closed'"; he did not say [anything] to other people crossing the bridge." Fourteen participants experienced microaggressions such as people frantically putting on their mask when passing by them and people glaring at them while grocery shopping. Two participants shared that they experienced racial discrimination on social media. Lastly, one encountered vandalism: "While driving, someone kept throwing garbage at my car window." 
Fig. 2 Distribution of responses for hypervigilance and nervousness during pandemic

\section{Hypervigilance and Nervousness during the Pandemic between Asians and non-Asians}

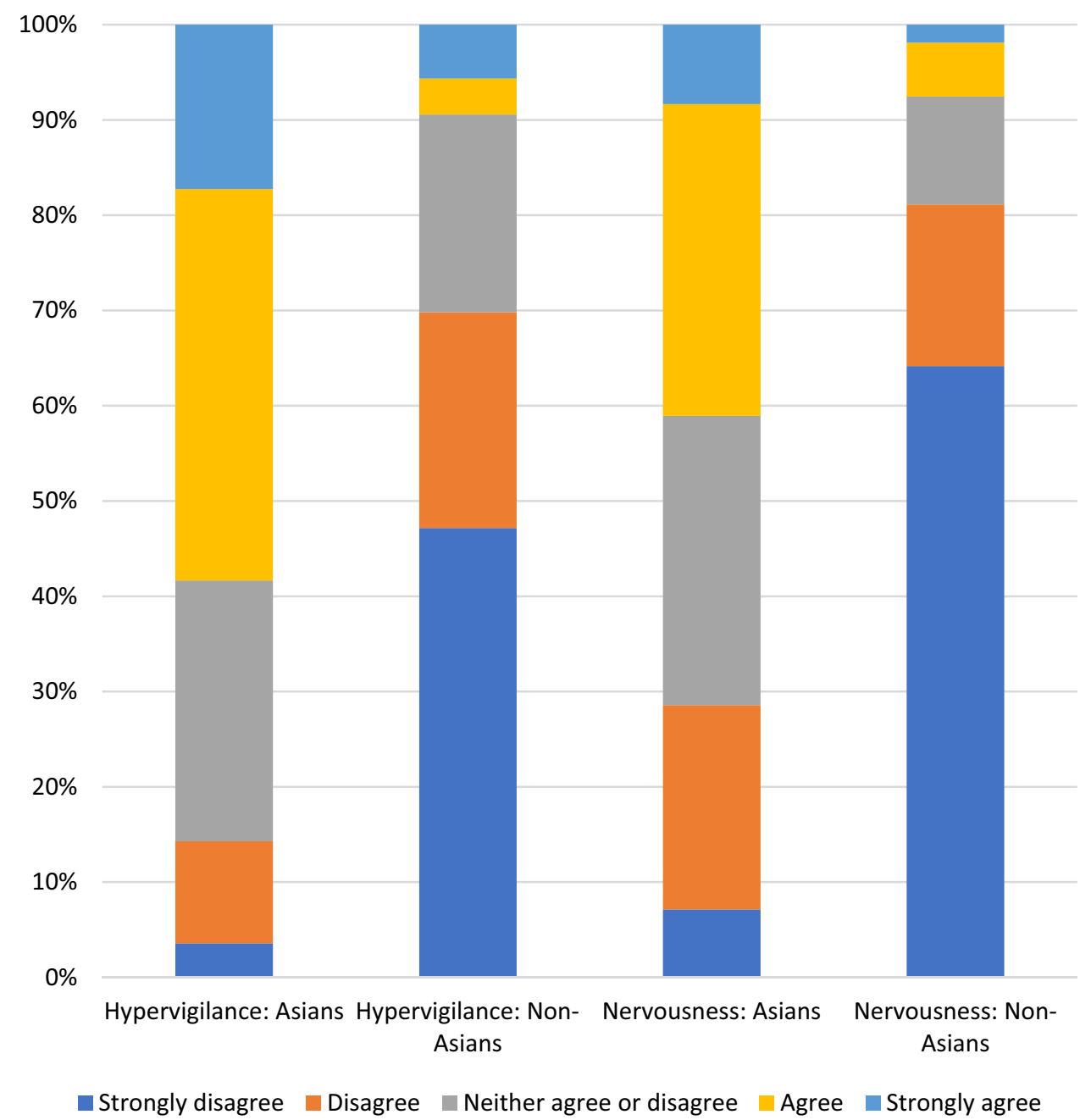

\section{Discussion}

Our study examined the impact of COVID-19 on experienced racial discrimination in small Asian communities. Our findings suggest that Asians were more likely to report experienced discrimination relative to non-Asians during the months of June and July 2020. Relative to non-Asians, our findings also suggest that Asians were more likely than non-Asians to report increased hypervigilance of their safety, nervousness in public, and anticipation of racial discrimination after the pandemic ends. Given that Asians in regions with minority Asian populations likely lack a strong cultural support network, these findings of disproportionate levels of discrimination are particularly concerning.

Experienced discrimination during COVID-19 can be detrimental to Asians because racial discrimination is associated with physical and mental health problems [29]. These associations were not assessed in-depth in this particular study. Future studies can use a mixed methods exploratory approach to identify health problems using a qualitative approach then assess the saliency of health problems using a quantitative approach. Previous research found that language and racial discrimination among Asian Americans are associated with a lowered sense of community support and chronic conditions, such as obesity, hypertension, heart attack, diabetes, and mental disorders $[8,30]$. This is concerning because respondents who completed the survey in an Asian language were more likely to report that they are nervous going outside than those who completed it in a nonAsian language. Given that self-reported racial discrimination among Asians nationwide is associated with anxiety and depressive disorders, and the general public has been experiencing higher scores of anxiety and depression compared to before COVID-19 [31, 32], Asians experience a double burden - the fear of discrimination on top of fear of COVID-19. These fears are further fueled by financial 
insecurities, as we found that $46.4 \%$ of our respondents indicated not working or working less during the pandemic than before. This may be related to how Asian businesses have been affected due to the COVID-19-related racial discrimination [33]. Such financial stress and insecurity have been associated with long-term poorer health and psychological distress $[34,35]$. Furthermore, fear of discrimination may deter Asians from accessing healthcare because scapegoated groups are often more reluctant to seek out medical care even when symptomatic, relative to those non scapegoated groups [26]. Therefore, in conjunction with a decreased likelihood of seeking out healthcare due to fear of mistreatment, discrimination could lead to longitudinal health consequences that disproportionately affect Asians in areas with smaller Asian populations.

Another matter of concern is how the discrimination may affect individuals' willingness to adhere to preventative measures for COVID-19. In June 2020, the World Health Organization (WHO) released guidelines on the proper use of masks to prevent the transmission of COVID-19 [36]. Despite this being a national guideline, over one-third of our respondents reported the cause of their experienced discrimination as mask-wearing and some respondents shared that they were verbally attacked for wearing a mask. Another national survey from June found that $36 \%$ of Asians worried that people would be suspicious of them if they wore a mask [37]. This is concerning because even though mask-wearing was a national recommendation, and a required mandate in some counties, Asians were assaulted and discriminated against for adhering to the guidelines [38]. Discrimination due to mask-wearing can potentially be attributed to the fact that face masks are less common in Western culture, while considered a hygiene etiquette in many Asian countries [39]. Further, this fear of discrimination may disincentivize individuals from testing for COVID-19 as a positive result would confirm the existing prejudices [40]. This may also prevent individuals from following Centers for Disease Control and Prevention's recommendations on maintaining a six feet social distance and avoiding large crowds [41]. Thus, fear of discrimination is associated with more high-risk behavior and fewer health-promoting activities [42], indicating that individuals may not wear their mask, avoid testing, and refuse to comply with social distancing rules in order to disassociate themselves from the stigma. Overall, discrimination against Asians for mask-wearing can exacerbate the transmission of the virus and should be addressed to curtail the COVID-19 pandemic.

Although less than $2 \%$ of reported discrimination in STOP AAPI Hate occurred in Florida [16], our findings demonstrate that Asians in Florida have experienced racial discrimination during the pandemic and anticipate continued or increased level of discrimination after the pandemic ends. This further accentuates that their experiences may be overlooked and underreported. Previous research has found that Chinese-Americans who lived in neighborhoods with no other Chinese-Americans were 3.45 times more likely to experience unfair treatment than those who lived within all-Chinese communities. Our findings also suggest that Asians in small Asian communities may experience more racial discrimination than those who live in communities with larger Asian populations. This difference in discrimination is likely attributed to being considered more 'foreign' or as an 'outsider' in places with smaller Asian populations, just as one of our respondents shared about entering an open community [18]. In addition, most of our Asian respondents reported few or no Asians in their community. The lack of cultural support may also lead to negative health consequences because social support can buffer the relationship between discrimination and health, especially considering how emotional support can be protective against illness [43]. Future multilevel studies can understand the mediating impact of community-level factors on discrimination-related experiences and health outcomes.

Our findings are consistent with the nationwide STOP AAPI Hate data collected between March 19 and March 25, 2020 [5]: verbal harassment and shunning were the two leading attributable causes of discrimination. The STOP AAPI Hate survey and the present study reported race as the primary reason for discrimination $(89.5 \%$ vs. $94.2 \%$, respectively). Our findings offer a unique perspective of the disproportionate discriminations and subsequent health risks that Asians in isolated communities face during the COVID-19 pandemic. This necessitates additional longitudinal research to understand the impact that hypervigilance, nervousness to go out into the community, and anticipated post-pandemic discrimination can have on health outcomes.

\section{Study Limitations}

Study limitations include recall and self-selection bias. Recall bias may occur if respondents are accustomed to experiencing discrimination, implying that these COVID-19 related experiences are not unique. Another limitation is the small size of the non-Asian participants and the lack of representation from other small minorities in Florida. Because we used respondent-driven sampling methods, there may be some sampling bias. There may have been self-selection bias because the sample was not randomly selected but recruited using respondent-driven sampling. Those who experienced discrimination may have participated in the survey because it is an opportunity to describe their experiences. Given that the survey was distributed via email and social media, those who are more active online will more likely complete the survey whereas others may delete the request. Additionally, Asians are a diverse group of different ethnicities who vary 
linguistically, culturally, and historically from each other [8]. Therefore, experiences of discrimination could be subjective and culturally different. We did not code the qualitative findings as these analyses will be performed for a future study: instead, we chose to include a few illustrative quotes for the present publication. Lastly, another study limitation is the lack of evaluation of interaction effects as well as the a priori selection of independent variables. However, our analysis does not show collinearity between the variables as the variable inflation factor were all less than 2.0 [44].

\section{New Contribution to the Literature}

To the best of our knowledge, this is the first study to examine experienced discrimination, hypervigilance, nervousness, and anticipated discrimination among Asians in Florida during the COVID-19 pandemic. Our findings indicate that small Asian communities in the U.S. experience a double burden: the fear of experiencing anti-Asian discrimination compounded with the fear of contracting COVID-19. Given that racial discrimination is intricately tied to severe physical and mental health outcomes among Asians, news agencies and health organizations should refrain from using discriminatory language (e.g. "China virus") and attributing the disease to a race/ethnicity. In addition, public health campaigns should use careful and non-accusatory language free of biases to promote healthy behaviors in future, similar instances.

\section{Data and/or Code Availability}

The datasets generated during and/or analyzed during the current study are available from the corresponding author on reasonable request.

Acknowledgements We would like to thank Dr. Sahnah Lim, Dr. Simona Kwon, and the 'Speak Up on Covid-19' team for giving us the tools and sharing Mt. Sinai's COVID-19 related survey measures (NYU Langone Health's Clinical and Translational Science Institute: UL1 TR001445; Mount Sinai Health System (MSHS) Translational Science Hub: UL1 TR00143305). We would like to thank our translators. Research reported in this publication was supported by the National Institute on Drug Abuse (awards \#K99DA041494, R00DA041494), National Institute on Minority Health and Health Disparities (awards \#P20MD002288, U54MD002266, \#F31MD015988), National Institute of Allergy and Infectious Diseases (award 350 \#P30AI050409), and the National Institute of Mental Health (awards \#R01MH100021, P30MH116867) of the National Institutes of Health. The content is solely the responsibility of the authors and does not necessarily represent the official views of the National Institute on Drug Abuse, the National Institute on Minority Health and Health Disparities, the National Institute of Allergy and Infectious Diseases, the National Institute of Mental Health or the National Institutes of Health.
Author Contributions All authors contributed to the study conception and design. Material preparation, data collection, and analysis were performed by MK, SL, YL, CHS, and MK. The first draft of the manuscript was written by $\mathrm{MK}$, and all authors commented on previous versions of the manuscript. All authors read and approved the final manuscript.

Funding This study was funded by National Institute on Drug Abuse (awards \#K99DA041494, R00DA041494), National Institute on Minority Health and Health Disparities (awards \#P20MD002288, U54MD002266, \#F31MD015988), National Institute of Allergy and Infectious Diseases (award 350 \#P30AI050409), and the National Institute of Mental Health (awards \#R01MH100021, P30MH116867) of the National Institutes of Health.

\section{Declarations}

Conflict of interest Authors have no relevant financial or non-financial interests to disclose. The authors also have no conflicts of interest to declare that are relevant to the content of the article.

Ethical Approval All procedures performed in studies involving human participants were in accordance with the ethical standards of the institutional research committee and with the 1964 Helsinki Declaration and its later amendments or comparable ethical standards. The study was approved by the Bioethics Committee of the University of Miami School of Medicine (No. 20200660).

Consent to Participate Informed consent was obtained from all individual participants included in the study.

\section{References}

1. Misra S, Le PD, Goldmann E, Yang LH. Psychological impact of anti-Asian stigma due to the COVID-19 pandemic: a call for research, practice, and policy responses. Psychol Trauma. 2020. https://doi.org/10.1037/tra0000821.

2. Gover A, Harper S, Langton L. Anti-Asian hate crime during the COVID-19 pandemic: exploring the reproduction of inequality. Am J Crim Justice. 2020;45:647.

3. Wen J, Aston J, Liu X, Ying T. Effects of misleading media coverage on public health crisis: a case of the 2019 novel coronavirus outbreak in China. Anatolia. 2020;31:331.

4. Kulkarni M, Choi C. STOP AAPI HATE receives over 1,100 incident reports of verbal harassment, shunning, and physical assault in two weeks. Los Angeles: Asian Pacific Policy \& Planning Council and Chinese for Affirmative Action; 2020.

5. Boboltz S. Stabbing of Asian American toddler and family deemed a hate crime: Report2020. https://www.huffpost.com/ entry/stabbing-of-asian-american-toddler-and-family-deemed-ahate-crime-report_n_5e84b65fc5b6871702a84c0b.

6. Kantamneni N. The impact of the COVID-19 pandemic on marginalized populations in the United States: a research agenda. J Vocat Behav. 2020;119:103439.

7. National Council of Asian Pacific Americans. Over 450 Civil Rights, social justice and labor organizations call on congress to denounce anti-Asian racism around COVID-19. 2020.

8. Yoo HC, Gee GC, Takeuchi D. Discrimination and health among Asian American immigrants: disentangling racial from language discrimination. Soc Sci Med. 2009;68:726-32. 
9. Sanders-Phillips K. Racial discrimination: a continuum of violence exposure for children of color. Clin Child Fam Psychol Rev. 2009;12:174-95.

10. Lockwood KG, Marsland AL, Matthews KA, Gianaros PJ. Perceived discrimination and cardiovascular health disparities: a multisystem review and health neuroscience perspective. Ann N Y Acad Sci. 2018;1428:170-207.

11. Ven CM. Experiences, coping styles and mental health of Muslims following 9/11. Social Cosmos. 2012;3:76-82.

12. Stangl AL, Earnshaw VA, Brakel WV, Simbayi LC, Barré I, Dovidio JF. The Health Stigma and Discrimination Framework: a global, crosscutting framework to inform research, intervention development, and policy on health-related stigmas. BMC Med. 2019. https://doi.org/10.1186/s12916-019-1271-3.

13. Quinn DM, Camacho G., Pan-Weisz B, Williams MK. Visible and concealable stigmatized identities and mental health: experiences of racial discrimination and anticipated stigma. Stigma Health. 2019.

14. Thornicroft G, Brohan E, Rose D, Sartorius N, Leese M, Group IS. Global pattern of experienced and anticipated discrimination against people with schizophrenia: a cross-sectional survey. Lancet. 2009;373:408-15.

15. Scheim AI, Bauer GR. The Intersectional Discrimination Index: development and validation of measures of self-reported enacted and anticipated discrimination for intercategorical analysis. Soc Sci Med. 2019;226:225-35.

16. Russell J, Nham K. Incidents of coronavirus-related discrimination: San Francisco State University Asian American Studies 2020.

17. U.S. Census Bureau: 2018 American Community Survey 1-Year Estimates 2018

18. Goto S, Gee G, Takeuchi D. Strangers still? The experience of discrimination among Chinese Americans. J Commun Psychol. 2002;30:211-24.

19. U.S. Department of Commerce: Asian Population Percentage by State: U.S. Census Bureau 2017.

20. Shulman ST, McAuley JB, Pachman LM, Miller ML, Ruschhaupt DG. Risk of coronary abnormalities due to Kawasaki disease in urban area with small Asian population. Am J Dis Child. 1987;141:420-5.

21. Pew Research Center: The Rise of Asian Americans. Washington, D.C. 2013.

22. Carter RT, Forsyth J. Reactions to racial discrimination: emotional stress and help-seeking behaviors. Psychol Trauma Theory Res Pract Policy. 2010;2:183-91.

23. Hwang WC, Goto $\mathrm{S}$. The impact of perceived racial discrimination on the mental health of Asian American and Latino college students. Cultur Divers Ethnic Minor Psychol. 2008;14:326-35.

24. Sadler GR, Lee HC, Lim RS, Fullerton J. Recruitment of hardto-reach population subgroups via adaptations of the snowball sampling strategy. Nurs Health Sci. 2010;12:369-74.

25. Florida for Florida. Data USA 2018. https://datausa.io/profile/geo/ florida/demographics/languages

26. Brislin RW. Back-translation for cross-cultural research. J Cross Cult Psychol. 1970;1(3):185-216.

27. Speak Up on COVID-19. Mount Sinai; 2020 [July 23, 2020]; https://www.speakuponcovid.org/.

28. Council APPP, Action CfA: Coronavirus Anti-AAPI Racism Incident Report. 2020 [July 23, 2020]; https://docs.google.com/ forms/d/e/1FAIpQLSefH2Mq_zowEGcB4h5r06BXX2aX3IBGfA ENmEZDXUktCfPEeQ/viewform.
29. Williams DR, Neighbors HW, Jackson JS. Racial/ethnic discrimination and health: findings from community studies. Am J Public Health. 2003;93:200-8.

30. Gee GC, Ro A, Gavin A, Takeuchi DT. Disentangling the effects of racial and weight discrimination on body mass index and obesity among Asian Americans. Am J Public Health. 2008;98:493-500.

31. Gee GC, Spencer M, Chen J, Yip T, Takeuchi DT. The association between self-reported racial discrimination and 12-month DSMIV mental disorders among Asian Americans nationwide. Soc Sci Med. 2007;64:1984-96.

32. Vindegaard N, Benros ME. COVID-19 pandemic and mental health consequences: Systematic review of the current evidence. Brain Behav Immun. 2020;89:531-42.

33. Roberts N. As COVID-19 spreads, Manhattan's Chinatown contemplates a bleak future. 2020. https://www.marketplace.org/ 2020/03/16/as-covid-19-spreads-manhattans-chinatown-conte mplates-a-bleak-future/.

34. Virtanen P, Vahtera J. VKivimäki M, Pentti J, Ferrie J. Employment security and health. J Epidemiol Commun Health. 2002;56:569-74.

35. Gutman A. Failing Economy. Failing Health: Harvard Public Health; 2014.

36. Advice on the use of masks in the context of COVID-19. Interim guidance 5 June 2020: World Health Organization (WHO); 2020.

37. Ruiz NG, Horowitz J, Tamir C. Many Black and Asian Americans say they have experienced discrimination amid the COVID-19 outbreak. Pew Research Center. 2020; https://www.pewsocialt rends.org/2020/07/01/many-black-and-asian-americans-say-theyhave-experienced-discrimination-amid-the-covid-19-outbreak/.

38. Brooks JT, Butler JC, Redfield RR. Universal masking to prevent SARS-CoV-2 transmission-the time is now. JAMA. 2020.

39. Wong SH, Teoh JYC, Leung $\mathrm{CH}$, Wu WKK, Yip BHK, Wong MCS. COVID-19 and public interest in face mask use. Am J Respir Crit Care Med. 2020.

40. Egelko A, Arnaout L, Garoon J, Streed C, Berger Z. "Do I have to be tested?": understanding reluctance to be screened for COVID19. Am J Public Health. 2020;110:1769-71.

41. Centers for Disease Control and Prevention: Social Distancing. Atlanta: Centers for Disease Control and Prevention; 2020 [updated November 17, 2020 cited 2020 December 6, 2020]. https://www.cdc.gov/coronavirus/2019-ncov/prevent-getting-sick/ social-distancing.html.

42. Williams DR, Lawrence JA, Davis BA, Vu C. Understanding how discrimination can affect health. Health Serv Res. 2019;54(Suppl 2):1374-88.

43. Gee GC, Chen J, Spencer MS, See S, Kuester OA, Tran D, Takeuchi D. Social support as a buffer for perceived unfair treatment among Filipino Americans: differences between San Francisco and Honolulu. Am J Public Health. 2006;96:677-84.

44. O'brien RM. A caution regarding rules of thumb for variance inflation factors. Qual Quant. 2007;41:673-90.

Publisher's Note Springer Nature remains neutral with regard to jurisdictional claims in published maps and institutional affiliations. 